\title{
CORRENTE INTERFERENCIAL NA DOR MUSCULAR DE INÍCIO TARDIA
}

\author{
Dayane Batista Franzes*, Jamile Irene Schäfer*, Vanessa Cristina Vieira*, Jhenifer Karvat**, \\ Alberito Rodrigo de Carvalho***, Gladson Ricardo Flor Bertolini**** \\ Autor correspondente: Gladson Ricardo Flor Bertolini - gladsonricardo@gmail.com \\ * Discente do curso de graduação em Fisioterapia da Universidade Estadual do Oeste do Paraná \\ ** Mestre em Biociências e Saúde pela Universidade Estadual do Oeste do Paraná \\ *** Mestre, professor Assistente do curso de Fisioterapia da Universidade Estadual do Oeste do Paraná \\ **** Doutor, professor Adjunto do curso de Fisioterapia e Mestrado em Biociências e Saúde da Universidade Estadual do \\ Oeste do Paraná
}

\section{Resumo}

Objetivo: avaliar o uso da corrente interferencial, sobre o tríceps sural de voluntários, com dor muscular de início tardio, por meio da escala visual analógica (EVA) e pela pressão local. Métodos: foram recrutados 14 indivíduos saudáveis e sedentários, os quais foram distribuídos aleatoriamente em Grupo Placebo e Grupo Experimental. Para avaliação da dor foi utilizada a EVA e um Algômetro de pressão, em 8 momentos $\left(A V_{1}-8\right)$ ao longo de 4 dias de intervenção $\left(1^{\circ}\right.$ dia exercício, $1^{\circ}-4^{\circ}$ dias eletroestimulação), sempre pré e pós-terapia. Visando promover a DMIT, os participantes realizaram movimentos concêntricos e excêntricos de planti e dorsiflexão sobre um degrau de $20 \mathrm{~cm}$ de altura. Para a realização da eletroestimulação, os eletrodos foram posicionados no ventre no músculo gastrocnêmio lateral e outro no ventre do músculo gastrocnêmio medial, sendo aplicada durante 20 minutos, em intensidade forte, porém confortável, com frequência base de $4 \mathrm{kHz}$, na forma bipolar e com frequência modulada pela amplitude (AMF) de $100 \mathrm{~Hz}$. Resultados: houve diferenças intragrupos, $A V_{1}$ do grupo placebo foi diferente de todas as seguintes, já para o grupo experimental $A V_{1}$ não foi diferente de AV8. Na comparação entre os grupos houve diferenças significativas: em AV3, AV4, AV6 e AV8. Na avaliação da dor à pressão houve diferença estatística intragrupo, apenas para o placebo, sendo que $\mathrm{AV}_{3}$ mostrou-se menor do que $A V_{7}$. Conclusão: a corrente interferencial foi eficaz para a redução da sensação de dor muscular de início tardio.

Palavras-chave: Mialgia Epidêmica; Medição da dor; Analgesia. 


\title{
INTERFERENTIAL CURRENT IN DELAYED ONSET OF MUSCLE SORENESS
}

\begin{abstract}
Aims: to evaluate the use of interferential current on the sural triceps volunteers with delayed onset muscle soreness, through the visual analogue scale (VAS) and the local pressure. Methods: fourteen healthy, sedentary individuals were recruited and randomly distributed in: Placebo Group and Experimental Group. To assess pain VAS and pressure algometry was used 8 times $\left(E V_{1}-8\right)$ in 4 days of intervention $\left(1^{\text {st }}\right.$ day exercise, $1^{\text {st }}-4^{\text {th }}$ days electrostimulation), always before and after the therapy. To promote the DOMS, participants performed concentric and eccentric movements of planti-dorsiflexion on a $20 \mathrm{~cm}$ step. To perform the electrical stimulation, the electrodes were placed in the lateral gastrocnemius muscle belly and another in the medial gastrocnemius. It was applied for 20 minutes at a high intensity, still comfortable for the subjects, with a $4 \mathrm{kHz}$ base frequency, bipolar and with $100 \mathrm{~Hz}$ AMF. Results: there were differences among the groups. The EV 1 placebo displayed results different from all the others groups'. Experimental group AV's results weren't different from AV8's. When compared, significative differences were found em $\mathrm{AV}_{3}, \mathrm{AV}_{4}, \mathrm{AV} 6$ and $A V 8$. In assessing the pressure pain intragroup statistical difference was found only for placebo. EV3's was lower than EV7's. Conclusion: The interferential current was effective to reduce the feeling of delayed onset muscle soreness.
\end{abstract}

Keywords: Pleurodynia, Epidemic; Pain measurement; Analgesia.

\section{INTRODUÇÃO}

Indivíduos que iniciam uma nova atividade física, ou que subitamente aumentam o volume e intensidades do exercício, desenvolvem a Dor Muscular de Início Tardio (DMIT); frequentemente associada com exercícios excêntricos. Geralmente aparece 12-24 horas após os exercícios, atingindo um pico ao redor de 48 horas; sendo que a resolução completa pode demorar até 10 dias. Apesar de não ser considerado um grande problema à saúde, os sintomas podem potencialmente causar riscos, como alterações na absorção de choque, perda de força e coordenação, com o surgimento de movimentos compensatórios. Há uma falta de consenso dos mecanismos que produzem a DMIT, sendo conjecturado como possíveis causas: danos a sarcômeros e tecido conectivo, processo inflamatório local, adaptações neurais e até causas psicossociais. ${ }^{(1-3)}$ Experi- mentos em animais têm apontado a ação de fatores neurotróficos, independentes do processo inflamatório, como produtores da hipernocicepção. (4)

A DMIT é alvo de diversos estudos, devido seu principal sintoma ser indesejado, mas também pelo prejuízo no desempenho de atletas, e desta forma a adaptação e readaptação aos estímulos utilizados no treinamento físico. ${ }^{(5)}$ Surgem várias opções de tratamento para a mesma, incluindo agentes farmacológicos antiinflamatórios e analgésicos, ${ }^{(5)}$ suplementação com fitoterápicos, ${ }^{(6)}$ crioterapia de corpo inteiro $^{(7)}$ e local, ${ }^{(8)}$ acupuntura, ${ }^{(9)}$ massagem, ${ }^{(1,10)}$ liberação miofascial, ${ }^{(11)}$ kinesio taping, ${ }^{(12)}$ alongamento muscular ${ }^{(13)}$ e recursos eletrotermoterapêuticos, como campo eletrostático pulsátil, ${ }^{(14)}$ laser de baixa potência, ultrassom terapêutico e corrente interferencial. ${ }^{(5)}$ 
A Corrente Interferencial ( $\mathrm{Cl})$ é uma técnica de estimulação elétrica, na qual estimula-se o tecido com correntes de média frequência, por meio de eletrodos colocados sobre a pele. Foi desenvolvido nos anos 50, visando diminuir a impedância e assim aumentar a profundidade de ação. Gera a frequência modulada pela amplitude (AMF), considerada de baixa frequência, e assim, no geral a corrente é capaz de penetrar mais profundamente mantendo bons efeitos analgésicos, ${ }^{(15)}$ sendo os mecanismos de ação atribuídos à "teoria das comportas", bloqueio de condução nervosa, aumento da circulação local, mecanismo central de supressão da dor e placebo. ${ }^{(16,17)}$

Contudo, visto que alguns estudos apontam resultados positivos $^{(18,19)}$ enquanto outro negativo ${ }^{(20)}$ sobre os efeitos da $\mathrm{Cl}$ em variáveis da DMIT, o presente estudo buscou avaliar o uso de uma técnica bipolar da $\mathrm{Cl}$, sobre o tríceps sural de voluntários, após realização de exercícios concêntricos e excêntricos analisando a dor pela escala visual analógica (EVA) e pela pressão local.

\section{MATERIAIS E MÉTODOS}

O presente estudo se classifica como quantitativo, de corte transversal, com amostra de conveniência, realizado após a aprovação pelo Comitê de Ética em Pesquisa (n. 056545/2015). Para tanto, foram recrutados 14 indivíduos saudáveis (sem queixas ou conhecimento de doenças), sedentários, com idade entre 20 e 26 anos, que nunca tivessem realizado qualquer tipo de eletroestimulação. Eram 5 homens e 9 mulheres, com idade de $21,8 \pm 1,8$, peso corporal de $70,3 \pm 10,9 \mathrm{~kg}$, altura $1,72 \pm 0,1 \mathrm{~m}$,

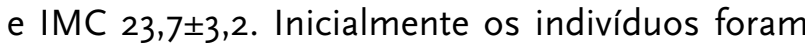
avaliados e distribuídos aleatoriamente, por meio de sorteio em envelope opaco, em 2 grupos: Grupo Placebo e Grupo Experimental. Cada voluntário foi considerado como duas amostras, pois foram avaliados isoladamente tanto o grupo muscular esquerdo como o direito, totalizando assim, 28 amostras, 14 em cada grupo.

\subsection{AVALIAÇÕES}

Utilizou-se para as mensurações a Escala Visual Analógica (EVA), com a finalidade de avaliar a intensidade da dor, e o Algômetro de pressão, visando analisar a pressão suportada até o início/agravamento do quadro álgico. Os indivíduos foram avaliados 8 vezes ao longo dos 4 dias de intervenção $\left(1^{\circ}\right.$ dia exercício, $1^{\circ}-4^{\circ}$ dias eletroestimulação), sempre pré e pós-terapia (ou simulação) (quadro 1).

Quadro 1 - Momentos de avaliação $(\mathrm{AV})$ e realização dos protocolos de DMIT e estimulação (marcados com x)

\begin{tabular}{|c|c|c|c|c|}
\hline & 10 DIA & 20 DIA & 30 DIA & 40 DIA \\
\hline Avaliação pré & AV1 & AV3 & AV5 & AV7 \\
\hline Exercício & $\mathrm{x}$ & & & $\mathrm{x}$ \\
\hline $\begin{array}{c}\text { Eletroestimulação / } \\
\text { placebo }\end{array}$ & $\mathrm{x}$ & $\mathrm{x}$ & $\mathrm{x}$ & AV8 \\
\hline Avaliação pós & $\mathrm{AV} 2$ & $\mathrm{AV} 4$ & $\mathrm{AV} 6$ & \\
\hline
\end{tabular}

$\mathrm{Na}$ EVA, o voluntário apontou em um escalímetro o nível de dor que sentia para cada membro individualmente, sem saber qual valor estava sendo indicado, pois no dispositivo uma régua de 10 centímetros, com indicação em milímetros, era visível apenas para o avaliador, sendo que ao participante era possível visualizar apenas o valor mínimo (sem dor) e máximo (máxima dor imaginável), respectivamente o e 10. 
O Algômetro de Pressão utilizado foi da marca Kratos ${ }^{\circledR}$ (São Paulo - SP - Brasil), com haste metálica e ponta achatada de $1 \mathrm{~cm}$ de diâmetro, pressionando na região medial do tríceps sural (entre os ventres dos gastrocnêmios) cerca de $10 \mathrm{~cm}$ abaixo da fossa poplítea, com pressão gradual, vertical, até o voluntário relatar dor. Após a mensuração, foi anotada a força (Kgf) necessária para ocorrer o estímulo doloroso em cada indivíduo.

\subsection{DMIT}

Visando promover a DMIT, os participantes realizaram movimentos concêntricos e excêntricos para o tríceps sural, realizando planti e dorsiflexões, respectivamente, em ortostatismo sobre um degrau de $20 \mathrm{~cm}$ de altura ( 3 séries de 20 repetições, adaptado de Bonfim et al. ${ }^{(13)}$ Não foi utilizada sobrecarga, desta forma, a carga foi dada apenas pelo peso do próprio corpo e pela ação da gravidade.

\subsection{ELETROESTIMULAÇÃO}

Para a realização da eletroestimulação, a parte posterior da perna foi dividida em terço superior, médio e inferior, sendo que os eletrodos foram posicionados entre o terço superior e médio, um deles no ventre no músculo gastrocnêmio lateral e outro no ventre do músculo gastrocnêmio medial. Os voluntários foram posicionados em decúbito ventral, e a corrente interferencial (Neurovector - Ibra$\operatorname{med} \circledast$, Amparo - SP - Brasil) foi aplicada, após o exercício, durante 20 minutos, em intensidade forte, porém confortável, com frequência base de 4 $\mathrm{kHz}$, na forma bipolar e com AMF de $100 \mathrm{~Hz}$, com eletrodos de borracha-silicone $\left(8 \mathrm{~cm}^{2}\right)$ e gel hidrossolúvel, em ambos os membros, sendo que prévio à eletroestimulação a pele era limpa com álcool $70^{\circ}$. Este procedimento foi repetido em 24, 48 e 72 horas após a realização do exercício gerador da DMIT.

Para o grupo placebo, os procedimentos adotados foram semelhantes, contudo, após os eletrodos serem posicionados sobre o grupo muscular, o aparelho não foi ligado, sendo explicado ao participante que a eletroestimulação ocorreria em nível subliminar, ou seja, não sentiriam nenhum tipo de parestesia com a aplicação da corrente.

\section{ANÁLISE DOS DADOS}

Tendo-se como base os dados da EVA, para um desvio-padrão de 3,0, diferença a ser detectada de 3,0, amostra de 14, nível de significância de $5 \%$, o poder do teste foi de $85 \%$. Os dados foram apresentados em média e desvio padrão. Para a comparação dos dados, da EVA e do Algômetro de Pressão, foram realizadas comparações intragrupos com uso de Anova medidas repetidas. Para comparar as avaliações entre os dois grupos foi utilizado Teste $t$ não pareado. Em ambos os casos o nível de significância aceito foi $5 \%$.

\section{RESULTADOS}

\subsection{EVA}

$\mathrm{Na}$ avaliação da sensação dolorosa foi possível observar que houve diferença estatística entre as medidas intragrupo, fato que ocorreu tanto no placebo $(F(7 ; 77)=14,2 ; p<0,001)$ quanto no experimental $(F(7 ; 77)=9,4 ; p<0,001)$. Para o placebo $A V_{1}$ foi diferente de todas as seguintes $\left(A V_{2}-p=0,009 ; A V_{3}\right.$ - $p=0,001 ; A V_{4}$ e $A V_{5}-p=0,002 ; A V 6-p=0,003$ $A V_{7}-p=0,011$; e $\left.A V 8-p=0,010\right)$; ainda $A V_{3}$ mostrou-se maior do que AV8 $(p=0,024)$. Para o grupo experimental houve diferenças entre $A V_{1}$ com as seguintes, exceto $A V 8\left(A V_{2}-p=0,035 ; A V_{3}-\right.$ $\mathrm{p}=0,003 ; A V_{4}$ e $A V_{5}-p<0,001 ; A V 6-p=0,001 ; A V_{7}$ - $p=0,041$; e $A V 8$ - $p=0,167)$, observou-se ainda que $\mathrm{AV}_{5}(\mathrm{p}=0,027)$ e $A V_{7}(\mathrm{p}=0,017)$ foram maiores do que AV8.

$\mathrm{Na}$ comparação entre os grupos, observando-se a primeira, segunda, quinta e sétima avaliações não houve diferenças significativas, sendo as diferenças para as respectivas avaliações: o\%, 38,5\%, 
$26 \%$ e $36 \%$. Porém, nas outras comparações houve diferenças significativas: $3^{\text {a }}$ (variação de $38 \%, p=$
$0,023) ; 4^{a}(33 \%, p=0,038) ; 6^{a}(46 \%, p=0,013)$ e $8^{a}$ $(52 \%, p=0,021)$.

Tabela 1 - Comparação dos valores da EVA obtidos nos 2 grupos, nos diferentes momentos de avaliação (AV)

\begin{tabular}{ccccccccc}
\hline & $\mathrm{AV} \mathbf{1}$ & $\mathrm{AV} \mathbf{2}$ & $\mathrm{AV}_{\mathbf{3}}{ }^{*}$ & $\mathrm{AV}_{\mathbf{4}}{ }^{*}$ & $\mathrm{AV} \mathbf{5}$ & $\mathrm{AV}_{\mathbf{6}}{ }^{*}$ & $\mathrm{AV} \mathbf{7}$ & $\mathrm{AV}_{\mathbf{8}}$ \\
\hline Placebo & $0.0 \pm \mathrm{O} . \mathrm{Oa}$ & $4.3 \pm 2.9 \mathrm{bc}$ & $6.3 \pm 3.2 \mathrm{~b}$ & $5.5 \pm 3 . \mathrm{Obc}$ & $5.8 \pm 3.3 \mathrm{bc}$ & $5.4 \pm 3.1 \mathrm{bc}$ & $4.8 \pm 3.3 \mathrm{bc}$ & $4.5 \pm 3.1 \mathrm{c}$ \\
Experimental & $0.0 \pm \mathrm{O} . \mathrm{Oa}$ & $2.7 \pm 2.1 \mathrm{bc}$ & $3.9 \pm 2.3 \mathrm{bc}$ & $3.7 \pm 1.6 \mathrm{bc}$ & $4.3 \pm 1.9 \mathrm{c}$ & $2.9 \pm 1.6 \mathrm{bc}$ & $3.1 \pm 2.5 \mathrm{c}$ & $2.2 \pm 2.2 \mathrm{ab}$ \\
\hline
\end{tabular}

Letras iguais correspondem a igualdades estatísticas dentro do grupo. *Diferenças estatísticas na comparação entre os grupos $p<0,05$.

\subsection{ALGÔMETRO DE PRESSÃO}

Na avaliação da dor à pressão foi possível observar que houve diferença estatística entre as medidas intragrupo, apenas para o placebo $(F(7 ; 77)=2,9$; $p=0,008)$, sendo que $A_{3}$ mostrou-se menor do que $A V_{7}(p=0,023)$.
$\mathrm{Na}$ comparação entre os grupos observou-se diferença nas seguintes avaliações: $1^{\mathrm{a}}$ (variação de $52 \%, p=0,02) ; 7^{\mathrm{a}}(36 \%, \mathrm{p}=0,001)$ e $8^{\mathrm{a}}(35 \%$, $\mathrm{p}=0,003)$, nas demais avaliações não houve diferenças significativas (tabela 2 ).

Tabela 2 - Comparação dos valores observados pelo Algômetro de pressão em Kgf, para os diferentes grupos, nos diferentes momentos de avaliação (AV)

\begin{tabular}{ccccccccc}
\hline & $\mathrm{AV}_{\mathbf{1}}{ }^{*}$ & $\mathrm{AV} \mathbf{2}$ & $\mathrm{AV} \mathbf{3}$ & $\mathrm{AV} \mathbf{4}$ & $\mathrm{AV} \mathbf{5}$ & $\mathrm{AV} \mathbf{6}$ & $\mathrm{AV} \mathbf{7}^{*}$ & $\mathrm{AV}_{\mathbf{8}}{ }^{*}$ \\
\hline Placebo & $12.8 \pm 4.7 \mathrm{ab}$ & $12.2 \pm 4.6 \mathrm{ab}$ & $10.5 \pm 3.7 \mathrm{a}$ & $9.5 \pm 3.4 \mathrm{ab}$ & $10.8 \pm 3.0 \mathrm{ab}$ & $10.6 \pm 3.5 \mathrm{ab}$ & $12.2 \pm 3.7 \mathrm{a}$ & $11.6 \pm 4.1 \mathrm{ab}$ \\
Experimental & $9.2 \pm 2.6 \mathrm{a}$ & $9.6 \pm 4.4 \mathrm{a}$ & $8.2 \pm 3.0 \mathrm{a}$ & $7.8 \pm 2.6 \mathrm{a}$ & $8.9 \pm 3.9 \mathrm{a}$ & $9.2 \pm 3.7 \mathrm{a}$ & $7.9 \pm 2.7 \mathrm{a}$ & $7.6 \pm 2.4 \mathrm{a}$ \\
\hline
\end{tabular}

Letras iguais correspondem a igualdades estatísticas dentro do grupo. *Diferenças estatísticas na comparação entre os grupos $p<0,05$

\section{DISCUSSÃO}

A contração excêntrica é um grande causador de DMIT, sendo que a intensidade do mesmo é um fator preponderante, causando lesões em estruturas musculoesqueléticas. ${ }^{(5)}$ No presente estudo utilizou-se uma adaptação do protocolo de Bonfim et al, (13) no qual são geradas contrações concêntricas e excêntricas do tríceps sural, sendo a carga gerada pelo próprio peso corporal. Tal adaptação mostrou-se útil na geração da DMIT pela VAS, contudo, o efeito não foi semelhante para alterar o limiar de dor à pressão.
No tecido muscular, apesar de polimodais, as fibras mais finas (tipo IV) são responsáveis pela transmissão da dor gerada por estímulos químicos, diferente daquela gerada por estímulos mecânicos (tipo III), (5) esta pode ser a causa dos achados citados anteriormente. Ainda, segundo Finocchietti, Graven-Nielsen e Arendt-Nielsen, ${ }^{(21)}$ visto que a dor muscular é frequentemente associada com distribuição heterogênea na avaliação mecânica, o algômetro de pressão estático, semelhante ao utilizado no presente estudo, apresenta dificuldades de avaliar a distribuição espacial e temporal.

Contudo, Rocha et al ${ }^{(22)}$ utilizando o algômetro de pressão para avaliar alterações na DMIT, após 
contrações excêntricas de flexo-extensão de joelho, utilizando a $\mathrm{Cl}$ com finalidades analgésicas, observaram que houve alteração no limiar do mesmo, porém, vale salientar que os autores realizaram estudo piloto e, desta forma, escolheram o ponto de maior dor na região central dos isquiotibiais. No presente estudo, optou-se por utilizar a região central do tríceps sural, mas, sem avaliação prévia (estudo piloto) visando analisar se este era o ponto mecanicamente mais sensível.

No presente estudo, a técnica escolhida para aplicação foi bipolar, em que a interferência ocorre dentro do equipamento (pré-moduladas), sendo esta outra limitação do presente estudo, pois nesta apresentação a voltagem, apesar de atingir tecidos profundos, é maior em linha com o circuito, ${ }^{(23)}$ ou seja, o efeito não ocorria em toda a região do tríceps sural. A técnica foi semelhante à realizada por Rocha et al, ${ }^{(22)}$ contudo, os autores utilizaram uma variação de AMF entre $80-150 \mathrm{~Hz}$, durante $30 \mathrm{mi}$ nutos, relatam que foi necessário elevar a intensidade em diversos momentos. No presente estudo optou-se pela fixação da AMF em $100 \mathrm{~Hz}$, visto que possivelmente o $\Delta \mathrm{f}$ não produz efeitos sobre o número ou limiar de acomodações. ${ }^{(24,25)}$

Apesar de serem aventadas na literatura, como possibilidades para o efeito analgésico, a "teoria das comportas", bloqueio de condução nervosa, aumento da circulação local, mecanismo central de supressão da dor e placebo, ${ }^{(16,17)}$ estudos em indivíduos saudáveis apresentam certa controvérsia com relação ao possível efeito de bloqueio de condução nervosa, ${ }^{(26-28)}$ sendo que talvez para obtenção de efeitos analgésicos seja necessária a presença de quadros álgicos já instalados, e não apenas alterações sobre os limiares de despolarização de fibras de dor. ${ }^{(29)}$ Conforme realizado no presente estudo, visto que não havia contração muscular (sem grandes aumentos de fluxo sanguíneo local), a intensidade utilizada era forte, porém confortável (possivelmente não havia estímulo para liberação de opioides) e houve diferenças com relação ao grupo placebo, a provável via de analgesia foi pela "teoria das comportas", principalmente pelo efeito temporário observado logo após a aplicação da terapia.

De forma contrária, Minder et al(20) avaliando os efeitos da Cl em DMIT (exercícios de flexão de cotovelo até a exaustão), por 30 minutos diários, 5 dias consecutivos. Não observaram efetividade da terapia, para o torque isométrico, ângulo de repouso, limiar à pressão ou EVA, com a variação de AMF baixa (10-20 Hz) ou alta $(80-150 \mathrm{~Hz})$. Já Schmitz et al,(18) para o mesmo grupo muscular, mas, utilizando técnica com posicionamento de 4 eletrodos, observaram que, após 48 horas do exercício excêntrico, a $\mathrm{Cl}$ aplicada por 30 minutos, tanto baixa $(10 \mathrm{~Hz})$ quanto em alta frequência $(100 \mathrm{~Hz})$, produzia redução do quadro álgico (avaliado pela EVA), sem alterações nos níveis de cortisol. Vale salientar que os parâmetros, dos estudos citados acima, diferem da presente pesquisa tanto no tempo total utilizado, padrão de avaliações, grupos musculares ou técnicas de posicionamento.

Zeng et al, ${ }^{(15)}$ em meta-análise, apresentam a $\mathrm{Cl}$ como efetiva em promover alívio da dor para indivíduos com osteoartrite de joelho. Fato observado, em ensaio clínico, por Eftekharsadat et al. ${ }^{\left({ }^{(30)}\right.}$ no qual relatam tanto redução da dor, quanto melhora da função em indivíduos com osteoartrite; e Atamaz et al ${ }^{\left({ }^{(1)}\right)}$ que observaram menor quantidade de ingestão de paracetamol para indivíduos com tal disfunção. Já Fuentes et al ${ }^{(17)}$ relatam que, para dor musculoesquelética, existem indícios que a corrente interferencial deve ser utilizada como complementar, sendo que a mesma parece não ter efeitos superiores ao placebo em curto ou longo prazos. Salienta-se que tal fato, é diferente do observado no presente estudo, pois analisando os dados da EVA, a $\mathrm{Cl}$ foi efetiva para redução da dor, uma vez que o grupo placebo apresentou diferenças entre a primeira com todas as avaliações seguintes, já para o grupo com tratamento efetivo a última avaliação não foi diferente do valor basal (zero). Ainda, ao comparar os grupos, foi possível visualizar que a eletroestimulação produziu menores intensidades em diversos momentos de avaliação. 
Observando-se algumas limitações do presente estudo, recomenda-se uma maior especificidade do local de avaliação do algômetro de pressão, visando avaliar o local exato de maior incômodo nos voluntários, a comparação das técnicas bi com tetrapolar, ainda um número maior de avaliações, com intervalos menores após o uso da corrente, podem especificar a duração dos efeitos $\mathrm{da} \mathrm{Cl}$.

\section{CONCLUSÃO}

Conclui-se com o estudo que a corrente interferencial foi eficaz para a redução da sensação de dor muscular de início tardio.

\section{REFERÊNCIAS}

1. Nelson N. Delayed onset muscle soreness: Is massage effective? J Bodyw Mov Ther. 2013;17(4):475-82. doi.org/10.1016/j. jbmt.2013.03.0O2

2. Tricoli V. Mecanismos envolvidos na etiologia da dor muscular tardia. Rev Bras Ciênc Mov. 2001;9(2):39-44.

3. Foschini D, Prestes J, Charro MA. Relação entre exercício físico, dano muscular e dor muscular de início tardio. Rev Bras Cineantropom Desempenho Hum. 2007;9(1):101-6.

4. Mizumura K, Taguchi T. Delayed onset muscle soreness: Involvement of neurotrophic factors. J Physiol Sci. 2016;66(1):43-52. doi.org/10.1007/ s12576-015-0397-O

5. Nascimento CR V, Arruda SFM, Bacurau RFP, Navarro F. Dor muscular tardia: etiologia e tratamento. Revista Brasileira de Prescrição e Fisiologia do Exercício. 2007;1(2):90-9.

6. Matsumura MD, Zavorsky GS, Smoliga JM. The effects of pre-exercise ginger supplementation on muscle damage and delayed onset muscle soreness. Phytother Res. 2015;29(6):887-93.

7. Costello JT, Baker PRA, Minett GM, Bieuzen F, Stewart IB, Bleakley C. Whole-body cryotherapy (extreme cold air exposure) for preventing and treating muscle soreness after exercise in adults. J Evid Based Med. 2016; Epub ahead.

8. Tomadon A, Ferreira ÁJM, Liberali M, Matera NF, Portolez JLM, Bertolini GRF. Crioterapia como fator interveniente na dor muscular de início tardio. Revista Brasileira de Prescrição e Fisiologia do Exercício. 2014;13(4):197-2O2.

9. Fleckenstein J, Niederer D, Auerbach K, Bernhörster M, Hübscher M, Vogt L, et al. No effect of acupuncture in the relief of Delayed-Onset Muscle Soreness: results of a randomized controlled trial. Clin J Sport Med. 2015; Epub ahead. Available from: http://www. scopus.com/inward/record.url?eid=2-s2.O84946430349\&partnerID=tZOtx3yl

1O. Visconti L, Capra G, Carta G, Forni C, Janin D. Effect of massage on DOMS in ultramarathon runners: A pilot study. J Bodyw Mov Ther. 2015;19(3):458-63.

11. Cheatham SW, Kolber MJ, Cain M, Lee M. The effects of self-myofascial release using a foam roll or roller massager on joint range of motion, muscle recovery, and performance: a systematic review. Int J Sport Phys Ther. 2015;1O(6):827-38.

12. Lee YS, Bae SH, Hwang JA, Kim KY. The effects of kinesio taping on architecture, strength and pain of muscles in delayed onset muscle soreness of biceps brachii. J Phys Ther Sci. 2015;27(2):457-9.

13. Bonfim $A E O$, de Ré D, Gaffuri J, Costa MMA, Portolez JLM, Bertolini GRF. Use of static stretching as an intervenient factor in delayed onset muscle soreness. Rev Bras Med Esporte. 2010;16(5):349-52.

14. Gatterer H, Peters P, Philippe M, Burtscher $M$. The effect of pulsating electrostatic field application on the development of delayed onset of muscle soreness (DOMS) symptoms after eccentric exercise. J Phys Ther Sci. 2015;27(10):3105-7.

15. Zeng C, Yang T, Deng Z, Yang Y, Zhang Y, Lei $G$. Electrical stimulation for pain relief in knee osteoarthritis: systematic review and network meta-analysis. Osteoarthr Cartil. 2015;23(2):189-2O2.

16. Artioli DP, Bertolini GRF. Corrente interferencial vetorial: aplicação, parâmetros e resultados. Rev Bras Clin Med. 2O12;10(1):51-6. 
17. Fuentes JP, Olivo SA, Magee DJ, Gross DP. Effectiveness of interferential current therapy in the management of musculoskeletal pain: a systematic review and meta-analysis. Phys Ther. 2010;90(9):1219-38.

18. Schmitz RJ, Martin DE, Perrin DH, Iranmanesh $A$, Rogol AD. Effect of interferential current on perceived pain and serum cortisol associated with delayed onset muscle soreness. J Sport Rehabil. 1997;6(1):30-7.

19. Rocha CS, Lanferdini FJ, Kolberg C, Silva MF, Vaz MA, Partata WA., et al. Interferential therapy effect on mechanical pain threshold and isometric torque after delayed onset muscle soreness induction in human hamstrings. J Sports Sci. 2O12;3O(8):733-42.

2O. Minder PM, Noble JG, Alves-Guerreiro J, Hill ID, Lowe AS, Walsh DM, et al. Interferential therapy: lack of effect upon experimentally induced delayed onset muscle soreness. Clin Physiol Funct Imaging. 2002;22(5):339-47.

21. Finocchietti S, Graven-Nielsen T, ArendtNielsen L. Dynamic mechanical assessment of muscle hyperalgesia in humans: The dynamic algometer. Pain Res Manag. 2015;2O(1):29-34.

22. Rocha CS, Lanferdini FJ, Kolberg C, Silva MF, Vaz MA, Partata WA, et al. Interferential therapy effect on mechanical pain threshold and isometric torque after delayed onset muscle soreness induction in human hamstrings. J Sports Sci. 2O12;30(8):733-42.

23. Beatti A, Rayner A, Chipchase L, Souvlis T. Penetration and spread of interferential current in cutaneous, subcutaneous and muscle tissues. Physiotherapy. 2011;97(4):319-26.

24. Pivetta KM, Bertolini GRF. .F efects on the interferential current accommodation in healthy subjects. Rev Bras Med Esporte. 2O12;18(5):330-2.

25. Guerra TEC, Bertolini GRF. Efeitos da variação da rampa de entrega do $\cdot F$ sobre a acomodação da corrente interferencial em mulheres saudáveis. Rev Dor. 2012;13(1):25-9.

26. Pereira GD, Cassolato KM, Lazarin PH, Canto TO, Portolez JLM, Bertolini GRF. Interferential current effect, $2000 \mathrm{~Hz}$, on the induced pain threshold. Rev Bras Med do Esporte. 2011;17(4):257-60.

27. Gross DP, Armijo-Olivo S, Magee DJ, Fuentes C J. A preliminary investigation into the effects of active interferential current therapy and placebo on pressure pain sensitivity: a random crossover placebo controlled study. Physiotherapy. 2011;97(4):291-301.

28. Venancio RC, Pelegrini S, Gomes DQ, Nakano EY, Liebano RE. Effects of carrier frequency of interferential current on pressure pain threshold and sensory comfort in humans. Arch Phys Med Rehabil. 2013;94(1):95-102. doi.org/10.1016/j. apmr.2012.08.2O4

29. Silva DDO, Spironello A, Andretta G, Broetto GS, Jaskowiak JL, Bertolini GRF. Corrente interferencial no limiar de dor induzida à pressão e ao frio, nas frequências de 2 kHz, 4 kHz e 8 kHz. ConScientiae Saúde. 2015;14(2):222-8.

30. Eftekharsadat B, Babaei-Ghazani A, Habibzadeh A, Kolahi B. Efficacy of action potential simulation and interferential therapy in the rehabilitation of patients with knee osteoarthritis. Ther Adv Musculoskel Dis. 2015;7(3):67-75.

31. Atamaz FC, Durmaz B, Baydar M, Demircioglu OY, lyiyapici A, Kuran B, et al. Comparison of the efficacy of transcutaneous electrical nerve stimulation, interferential currents, and shortwave diathermy in knee osteoarthritis: A double-blind, randomized, controlled, multicenter study. Arch Phys Med Rehabil. 2012;93(5):748-56. doi.org/10.1016/j. apmr.2011.11.037 